\title{
Tamoxifen-Related Endometrial Disorder
}

National Cancer Institute

\section{Source}

National Cancer Institute. Tamoxifen-Related Endometrial Disorder. NCI Thesaurus. Code C40159.

A spectrum of endometrial abnormalities that occur in women who use tamoxifen to treat or prevent the development of breast cancer. These abnormalities include endometrial polyps, endometrial hyperplasia, and endometrial carcinoma. 